\title{
Development and characterisation of a self-powered measurement buoy prototype by means of piezoelectric energy harvester for monitoring activities in a marine environment
}

\author{
Damiano Alizzio ${ }^{1}$, Antonino Quattrocchi ${ }^{1}$, Roberto Montanini ${ }^{1}$ \\ ${ }^{1}$ Department of Engineering, University of Messina, C.da di Dio, Vill. S. Agata, 98166, Messina, Italy
}

\begin{abstract}
In the interest of our society, for example in Smart City but also in other specific backgrounds, environmental monitoring is an essential activity to measure the quality of different ecosystems. In fact, the need to obtain accurate and extended measurements in space and time has considerably become relevant. In very large environments, such as marine ones, technological solutions are required for the use of smart, automatic, and self-powered devices in order to reduce human maintenance service. This work presents a simple and innovative layout for a small self-powered floating buoy, with the aim of measuring and transmitting the detected data for visualization, storage and/or elaboration. The power supply was obtained using a cantilever harvester, based on piezoelectric patches, converting the motion of ripple waves. Such type of waves is characterized by frequencies between $1.50 \mathrm{~Hz}$ and $2.50 \mathrm{~Hz}$ with oscillation between $5.0^{\circ}$ and $7.0^{\circ}$. Specifically, a dedicated experimental setup was created to simulate the motion of ripple waves and to evaluate the suitability of the proposed design and the performance of the used harvester. Furthermore, a dynamic analytical model for the harvester has been defined and the uncertainty correlated to the harvested power has been evaluated. Finally, the harvested voltage and power have shown how the presented buoy behaves like a frequency transformer. Hence, although the used cantilever harvester does not work in its resonant frequency, the harvested electricity undergoes a significant increase.
\end{abstract}

\section{Section: RESEARCH PAPER}

Keywords: Piezoelectric patch; ripples waves; uncertainty estimation; motion frequency transformer

Citation: Damiano Alizzio, Antonino Quattrocchi, Roberto Montanini, Development and characterization of a self-powered measurement buoy prototype by means of piezoelectric energy harvester for monitoring activities in a marine environment, Acta IMEKO, vol. 10, no. 4, article 31, December 2021, identifier: IMEKO-ACTA-10 (2021)-04-31

Section Editor: Roberto Montanini, Università di Messina and Alfredo Cigada, Politecnico di Milano, Italy

Received September 10, 2021; In final form December 11, 2021; Published December 2021

Copyright: This is an open-access article distributed under the terms of the Creative Commons Attribution 3.0 License, which permits unrestricted use, distribution, and reproduction in any medium, provided the original author and source are credited.

Corresponding author: Damiano Alizzio, e-mail: damiano.alizzio@unime.it

\section{INTRODUCTION}

The purposes of monitoring procedures for marine environment are multiple and useful both for measuring the quality of the specific ecosystem and for estimating of the global anthropogenic impact, also in the field of Smart City [1], [2]. Most widespread activities range from detection of pollutants to meteorological or climatic measurements [3]. However, other aims concern the use of marine sensors for safety evaluation in coastal areas and protection from seismic events or biological hazards [4]. Scientific literature already presents technological solutions in the field of floating measurement devices with specialized sensors and data communication systems for different applications [5].

Measurement buoys provide a reading service for chemical and/or physical quantities through sensors and low energy electronics and guarantee a stable communication to the ground through suitable data transfer channels (WI-FI, Bluetooth, GSM) [6], [7]. Although a modest power input is foreseen for supplying the on-board instrumentation, the need to make these devices energy self-sufficient remains open. For such purpose, technologies are employed for ensuring a buoys independence from human presence and a constant source of electricity [8], [9].

Traditionally, measurement buoys are powered by photovoltaic systems and by wind turbines. Alippi et al. [10], Albaladejo et al. [11] and Hormann et al. [12] employed different 
size photovoltaic cells to feed sensor buoy systems for sea trials. In these studies, the main limitations were found in the dependence of the electricity production by the sun, by lacking in bad weather conditions and by inactivity during the night, and in the need to equip the measurement buoys with bulky and heavy systems and batteries. Instead, for wind turbines, the meteorological conditions are not optimal on the sea surface. In fact, the wind speed at $1 \mathrm{~m}$ above the sea level is a third of that at $80 \mathrm{~m}$. This results in a $73 \%$ reduction in wind power [13]. Recently, new energy sources, such as tidal currents and sea or ocean waves, have been investigated. Trevathan et al. [14] proposed tidal currents to supply sensors for marine measurements and compared the performances of different types of wind turbines. However, they concluded that such devices may not be cost-effective and risk-prone to apply due to biofouling and entanglement from drifting algae and sea grass wrack. Sea or ocean waves represent an attractive renewable source, indirectly related to both the sun and the wind, and with a high energy density [15]. Pelc et al. [16] stated that wave energy is vast and more reliable than most renewable sources. Furthermore, they highlighted that such energy at a given site is available up to $90 \%$ of the time, while photovoltaic and wind energy tend to be available only $20-30 \%$ of the time.

The adoption of an energy harvester by piezoelectric transducers $(\mathrm{PEH})$ is an advantageous solution to convert wave motion in electric energy. $\mathrm{PEH}$ are a well-known technique in literature. For example, Bolzea et al. [17] used such devices in cantilever configuration, demonstrating that the maximum power output is generated when the mechanical resonance is reached. Toyabur et al. [18] designed a multimode PEH, consisting of four elements, connected in parallel and in a cantilever configuration, to achieve different low frequency resonance modes $(10-20 \mathrm{~Hz})$. The authors showed that their system generates about four times more power than a single PEH. Pradeesh et al. [19] analysed, both experimentally and numerically, the effect of a proof mass fixed to a $\mathrm{PEH}$ in a cantilever configuration. The researchers obtained the best results as the proof mass was glued close to the clamped end. These works highlight the importance of a correct design for a cantilever type PEH, but they do not discuss the effect of the proof mass (i. e. the resonant frequency variation of the $\mathrm{PEH}$ ) on the harvested power. Recently, Montanini et al. [20] developed a PEH, using a glass fibre reinforced beam support. The authors studied the correlation between the mechanical working frequency and the harvested electrical power, analysing the deflection shape of the PEH under operating conditions by means of a scanning laser-Doppler vibrometer. After that, they investigated the conversion efficiency of this PEH [21] and the applicability of a low-power single-stage converter, able to automatically follow the changes in the resistive component of the output impedance, in order to maximize the energy yield [22], [23].

In the field of power supply for measurement buoys, PEHs have been sporadically applied due to the low frequency of the sea and ocean waves. Wu et al. [24] developed a PEH fixed to a floating buoy, that was anchored to the ocean floor. This device consisted of several cantilevers, on which many piezoelectric patches (PCs) were attached. The authors analyzed the size effect of the float and derived a numerical model to calculate the harvested energy. The research findings show that up to $24 \mathrm{~W}$ electric power can be generated with the piezoelectric cantilevers length of $1 \mathrm{~m}$ and the length of the buoy of $20 \mathrm{~m}$. Nabavi et al. [25] proposed the design of a beam-to-column piezoelectric

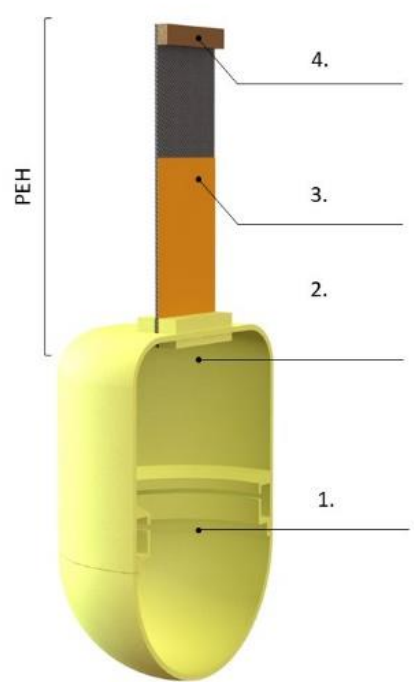

Figure 1. Concept design of the measurement floating buoy prototype. 1. $=$ hemispherical part, $2 .=$ cylindrical part, 3. $=$ PC and $4 .=$ proof mass.

system, able to power a large floating and instrumented ocean buoy. They derived and experimentally verified the equations of the electromechanical behavior of the device, demonstrating that the height amplitude and the low frequency of the wave guarantee the best performance. Additionally, using a baffled water tank, they developed a self-tuning buoy, which works based on the frequency of ocean waves. Recently, Alizzio et al. [26], [27] proposed an instrumented spar and fixed-point buoy, equipped with a $\mathrm{PEH}$, to convert the energy of the wave motion into electricity through some PCs, glued on deformable and floating bands. The buoy was designed, numerically simulated, and experimentally verified, obtaining a light structure able to self-power its on-board sensors and carrying out data transmission.

In this paper, the performance of a simple and innovative layout for a small measurement buoy, supplied by a cantilever $\mathrm{PEH}$, has been studied. The structure has been designed to convert the motion of the ripple waves in a cyclic oscillation with harmonics of high frequency, to which the PEH is subjected thanks to a suitable proof mass. In this context, a dedicated experimental setup has been implemented to simulate the motion of the ripple waves and to evaluate its effect on the electric response of the PEH. Such motion has been discretized on amplitude and frequency configurations, characterizing the dynamics of the proposed buoy with an analytical model of the PEH. Finally, the harvested power has been estimated and the related uncertainty has been evaluated.

\section{MATERIALS AND METHODS}

\subsection{Prototype of the measurement floating buoy}

The presented buoy prototype (Figure 1) was made of a floating structure, manufactured with a 3D printer using a highly durable photo-polymeric resin. It was divided in two pieces: the bottom one was designed with a hemispherical shape for an appropriate matching with the sea waves, while the upper one had a cylindrical shape where the PEH was set by a fixed joint. The two parts were connected by means of a thread, to hermetically contain a dedicated measurement instrumentation for monitoring the marine environment with a data transmission system. This PEH allowed to convert the alternative rotational (rolling) motion of the buoy, while this one was subject to ripple 


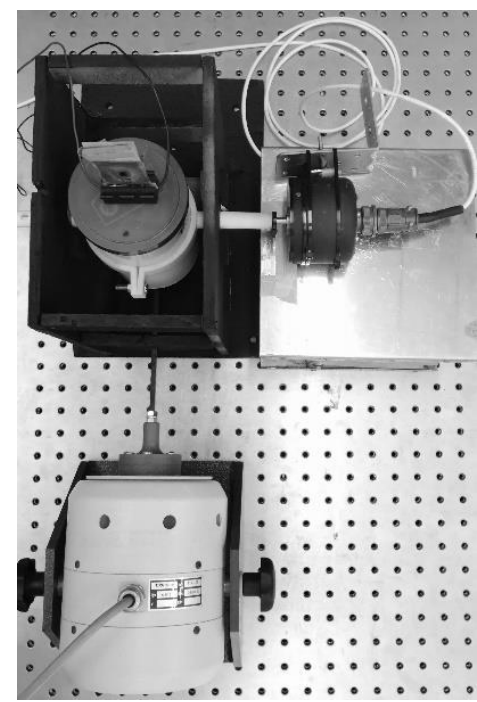

a)

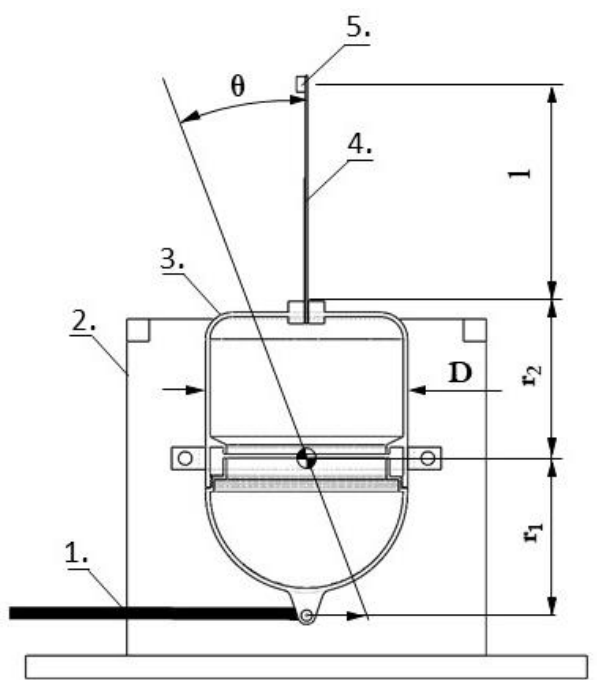

b)

Figure 2. a) image of the experimental setup and b) schema of the conversion system for the linear motion of the shaker in the imposed rolling motion to the buoy. $1 .=$ stinger of the shaker, $2 .=$ frame, 3. = body of the buoy, 4. = PEH, 5. = proof mass, $r_{1}=$ height of the hemispherical part of the buoy, $r_{2}=$ height of the cylindrical part of the buoy, $I=$ arm of the $\mathrm{PEH}, D=$ diameter of the buoy and $\vartheta=$ angular displacement.

waves, into electrical energy in order to provide an adequate power to supply all electronic devices within the same buoy.

\subsection{Piezoelectric energy harvester (PEH)}

The used PEH consisted of a cantilever support, on which a PC and a proof mass were glued at the opposite ends. The PC (DuraAct P-876 A.12, Physik Instrumente $\mathrm{GmbH}$ ) has sizes of $61.0 \mathrm{~mm} \times 35.0 \mathrm{~mm} \times 0.5 \mathrm{~mm}$ and an electrical capacity of 90 $\mathrm{nF}$. The active element of the PC, a thin layer of piezoceramic PIC255 powder, is encapsulated in a Kapton case. The PC shows a symmetrical structure; if it is deformed, the same amount of voltage is generated, opposite in charge, on the two surfaces of the electrodes. These types of devices have the double advantage of being also used as an actuator. When driven by an alternating voltage, the PCs act with a multiaxial deformation which depends on the amplitude and frequency of the power signal [28], [29]. The cantilever support, measuring $105 \mathrm{~mm} \times 35 \mathrm{~mm} \times 1 \mathrm{~mm}$, was made by manual layering of three layers of $0 \circ / 90^{\circ}$ oriented glass fibre and epoxy resin.

\subsection{Experimental setup and procedure}

The experimental setup (Figure 2a) included the buoy with its $\mathrm{PEH}$ and an electrodynamic shaker (mod. S 513, Tira), driven by a power amplifier (mod. BAA 120, Tira) and a function generator (mod. 33220 A, Agilent). In order to simulate the motion of the ripple waves in amplitude and frequency, a conversion system (Figure 2b) of the linear motion of the shaker was implemented. It consisted of a frame with two flanges, able to rotate the buoy around a fixed horizontal axis by means of two bearings. The imposed rolling motion was applied by connecting the stinger of the shaker to the bottom of the buoy (i. e. the vertex of the hemisphere) using a ball joint. The geometry of the conversion system is reported in Table 1 . The imposed rolling motion to the buoy was monitored by a rotational transducer (mod. 0600-0000,

Table 1. Geometric characteristics of the conversion system for the linear motion of the shaker in the imposed rolling motion to the buoy.

\begin{tabular}{ccccc}
\hline $\begin{array}{c}\text { Geometric } \\
\text { parameters }\end{array}$ & $r_{1}$ in $\mathbf{~ m m}$ & $r_{2}$ in $\mathbf{~ m m}$ & $\boldsymbol{l}$ in $\mathbf{~ m m}$ & $\boldsymbol{D}$ in $\mathbf{~ m m}$ \\
\hline & 70 & 70 & 100 & 90 \\
\hline
\end{tabular}

TRANS-TEK), set in the fixed horizontal axis of the two flanges. An oscilloscope (mod. TDS 5054B, Tektronix) was employed to measure the previous oscillation signal and the voltage response of the PEH on a resistive load of $100 \mathrm{k} \Omega$. Specifically, this resistive load was chosen in accordance with the maximum power transfer theorem, knowing the internal impedance of the PC [20].

The behaviour of the buoy was studied by varying the working frequency, the amplitude of the imposed rolling motion and the proof mass glued at the end of the cantilever support. The frequencies and amplitudes of the ripple waves were appropriately following the results of [27]. Therefore, the sinusoidal functions of the imposed rolling motion to the buoy were chosen with the characteristics shown in Table 2. The acquisition frequency of the signals was set at $2 \mathrm{kHz}$ and each test was repeated 5 times for every combination of working frequency $f_{w}$ and angular displacement $\theta$.

\subsection{Simplified model of the mechanical behaviour of the PEH}

The mechanical behavior of the analyzed PEH (i.e. a clamped-free beam with a proof mass) can be explained by a single degree of freedom (SDOF) model (Figure 3), according to [30]-[32].

For a $\mathrm{PEH}$ without a proof mass, the equation of motion of Euler-Bernoulli beam for undamped free vibrations can be considered:

$$
E I \frac{\partial^{4} w(x, t)}{\partial x^{4}}+m \frac{\partial^{2} w(x, t)}{\partial t^{2}}=0
$$

where $m, E$ and $I$ indicate the mass, the Young's modulus and the inertia momentum of the PEH, respectively. $w(x, t)$ is the absolute motion of the PEH along its axis expressed as:

$$
w(x, t)=w_{\text {rel }}(x, t)+w_{b}(x, t),
$$

Table 2. Characteristics of the imposed rolling motion to the buoy.

\begin{tabular}{lcccccc}
\hline & Case 1 & Case 2 & Case 3 & Case 4 & Case 5 \\
\hline Working frequency $\boldsymbol{f}_{\boldsymbol{w}}$ in $\mathrm{Hz}$ & 1.50 & 1.75 & 2.00 & 2.25 & 2.50 \\
Angular displacement $\boldsymbol{\vartheta}$ in & & 5.0 & 5.3 & 5.9 & 6.6 & 7.0 \\
\hline
\end{tabular}




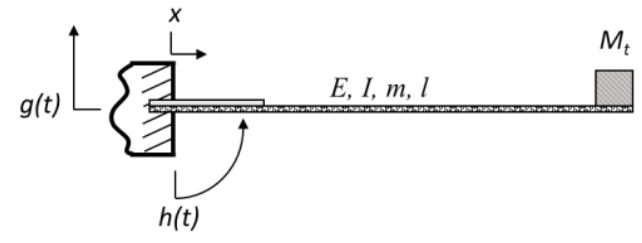

Figure 3. Scheme of the model for the PEH.
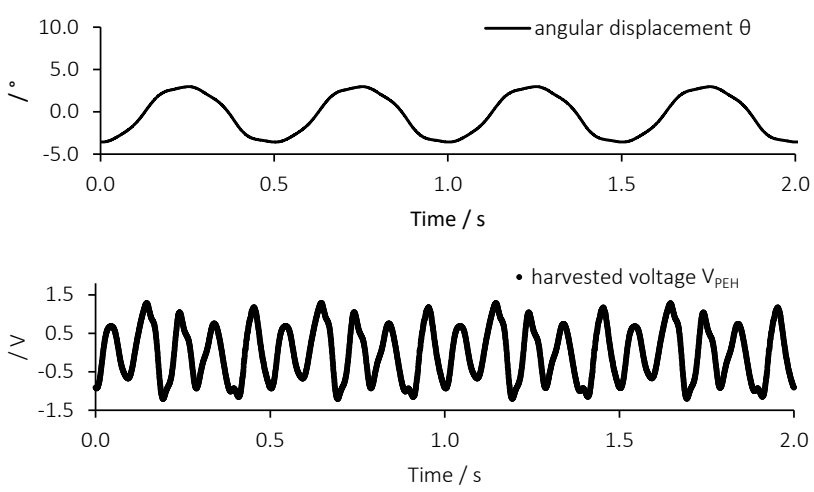

Figure 4. Typical signal of the angular displacement $\vartheta$ of the buoy (on the top) and of the harvested voltage $V_{P E H}$ by the PEH (on the bottom) at $2.00 \mathrm{~Hz}$.

where $w_{\text {rel }}(x, t)$ is the displacement, relative to the clamped end, and $w_{b}(x, t)$ is the absolute displacement of the buoy.

By introducing a proof mass and according to equation (2), equation (1) takes the form reported as follows:

$$
\begin{aligned}
E I \frac{\partial^{4}}{\partial x^{4}} w_{r e l}(x, t)+m \frac{\partial^{2}}{\partial t^{2}} w_{r e l}(x, t) & \\
= & -\left[m+(x-l) M_{t}\right] \frac{\partial^{2}}{\partial t^{2}} w_{b}(x, t),
\end{aligned}
$$

where $M_{t}$ denotes the proof mass and produces a new contribution.

$w_{b}(x, t)$ consists of the composition of an orthogonal translation $g(t)$ and a rotation $h(t)$ of the PEH clamped end:

$$
w_{b}(x, t)=\delta_{1}(x) g(t)+\delta_{2}(x) h(t) .
$$

In our case (i.e. clamped-free beam and Figure 2b), $\delta_{1}(x)=$ $1, \delta_{2}(x)=r_{2}+x, g(t)=r_{2} \sin \theta(t)$ and $h(t)=\theta(t)$, and equation (5) can be reported as:

$$
w_{b}(x, t)=r_{2} \sin \theta(t)+\left(r_{2}+x\right) \theta(t) .
$$

\subsection{Estimation of the harvested power by the PEH}

The harvested power by the PEH was evaluated according to the model proposed by Shu et al. [33], using equation (6):

$$
P=\frac{\pi}{\omega} \frac{V^{2}}{8 R}
$$

where $V$ and $\omega$ are respectively the amplitude and the pulsation of the harvested voltage and $R$ is the resistive load wired to the harvester.

Considering the complex trend of the harvested voltage $V_{P E H}$ of the PEH, $V$ was estimated as a specific average voltage, indicated with $\bar{V}$. It is the accumulated voltage inside the excitation period by the integral average of the rectified signal in equation (7):

$$
\bar{V}=\frac{1}{T} \int_{0}^{T}\left|V_{P E H}(t)\right| d t,
$$

where $T=1 / f_{w}$ is the excitation period and $f_{w}$ is the working frequency of the imposed rolling motion of the buoy.

For discrete signals, the equation (7) becomes:

$$
\bar{V}=\frac{1}{T} \sum_{t=0}^{T}\left|V_{P E H}(t)\right| .
$$

Hence, equation (6) takes the form of equation (9):

$$
\begin{gathered}
\bar{P}=\frac{1}{16 R T}\left(\sum_{t=0}^{T}\left|V_{P E H}(t)\right|\right)^{2}= \\
=\frac{f_{w}}{16 R}\left(\sum_{t=0}^{1 / f_{w}}\left|V_{P E H}(t)\right|\right)^{2},
\end{gathered}
$$

where $\bar{P}$ is the specific power, harvested by the PEH. It is here mentioned as specific power, hence it is a complex function of the working frequency due to its dependence on $\bar{V}$.

\section{RESULTS}

\subsection{Effects of the mechanical frequency}

Figure 4 shows the typical signals concerning the angular displacement $\theta$ of the buoy, measured on the fixed horizontal axis, and the harvested voltage $V_{P E H}$ by the PEH, following the imposed rolling motion. In this application the mechanical operating conditions of the $\mathrm{PEH}$ are quite different from those reported in the literature [24]-[27]. Indeed, the piezoelectric component is mechanically stressed by a non-inertial force field in which the motion is alternative. The working frequencies $f_{w}$ of the presented PEH are significantly lower than the typical ones of this devices, while the amplitude of the angular displacement $\theta$ does not allow the hypothesis of small oscillations. In Figure 4, although a certain period can be identifiable, the two acquired signals do not have the same dynamics. The angular displacement $\theta$ has a sufficiently sinusoidal behavior, according to the imposed rolling motion, while the harvested voltage $V_{P E H}$ is oscillating with a variable amplitude.

Figure 5 reports the magnitude of the Discrete Fourier Transform (DFT) of the signals of Figure 4, computed using a resolution of $0.10 \mathrm{~Hz}$. The imposed rolling motion is characterized by a single frequency at $2.00 \mathrm{~Hz}$, vice versa the harvested voltage $V_{P E H}$ has some harmonic components with higher order and with maximum amplitude at $10.00 \mathrm{~Hz}$. The identified phenomenon occurs for all the conducted tests and denotes a multimodal enhancement of the PEH. It must be pointed out that, although the employed shaker ensures a linear trend in the frequency range from $2 \mathrm{~Hz}$ to $7000 \mathrm{~Hz}$, its operation at the lower frequency limit remains optimal. In fact, there are no distortions since the amplitudes of the DFT of the angular displacement $\theta$, reported in Figure 5, do not show significant higher order harmonics.

Figure 6 illustrates the comparison between the magnitudes of the DFTs of the harvested voltage $V_{P E H}$ by varying the proof mass, glued to the free end of the $\mathrm{PEH}$, and following an angular displacement $\theta$ of the buoy of $6.6^{\circ}$ amplitude at a working frequency of $2.00 \mathrm{~Hz}$. A different applied proof mass (i. e. the resonant frequency of the PEH [20], [21]) does not cause a 

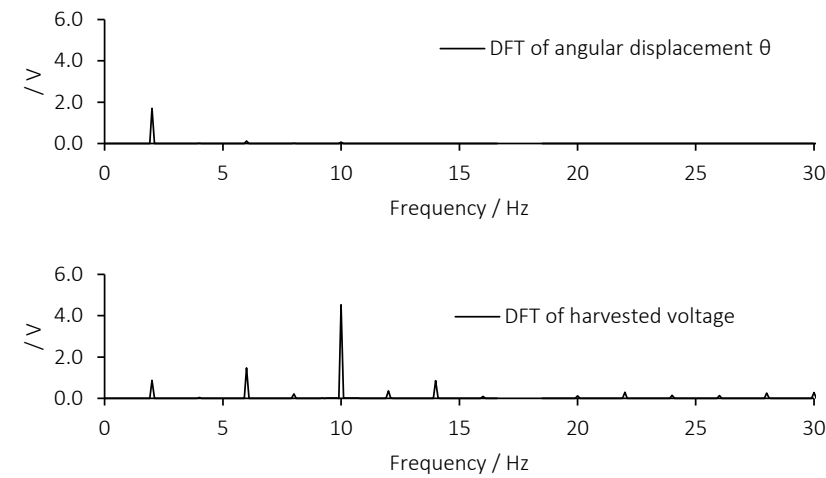

Figure 5. Magnitude of the DFT of the angular displacement $\vartheta$ (on the top) and of the harvested voltage $V_{P E H}$ (on the bottom) with a working frequency $f_{w}$ of $2.00 \mathrm{~Hz}$.

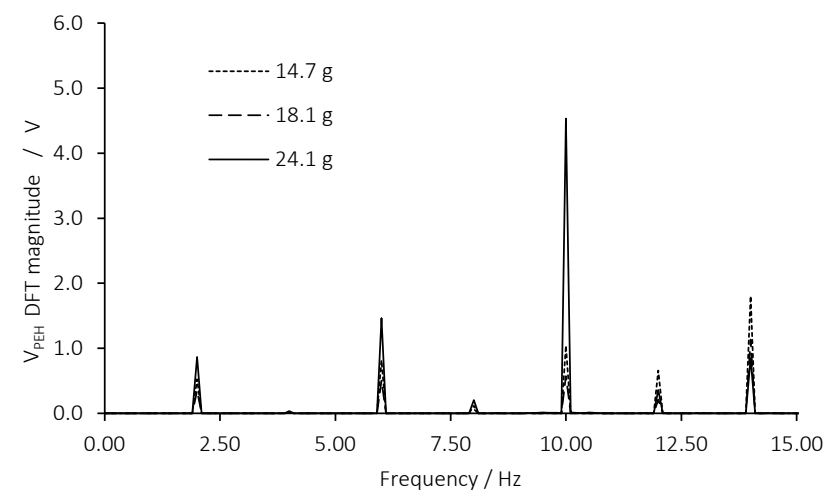

Figure 6. Comparison between the magnitude of the DFTs of the harvested voltage $V_{P E H}$ by varying the proof mass at an angular displacement $\vartheta$ of the buoy of $6.6^{\circ}$ at a working frequency $f_{w}$ of $2.00 \mathrm{~Hz}$.

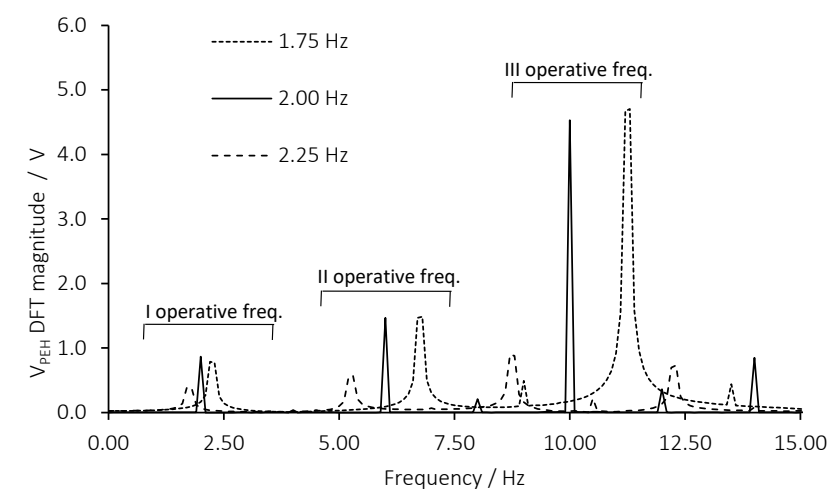

Figure 7. Comparison between the magnitudes of the DFTs of the harvested voltage $V_{P E H}$ by varying the imposed rolling motion at an angular displacement $\vartheta$ of the buoy of $6.6^{\circ}$ and using a proof mass of $24.1 \mathrm{~g}$.

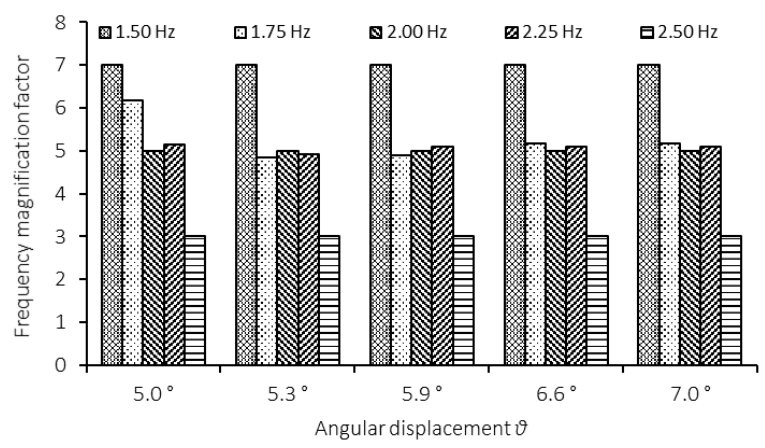

Figure 8. Comparison of the frequency magnification factors at different angular displacement $\vartheta$ of the imposed rolling motion and using a proof mass of $24.1 \mathrm{~g}$.

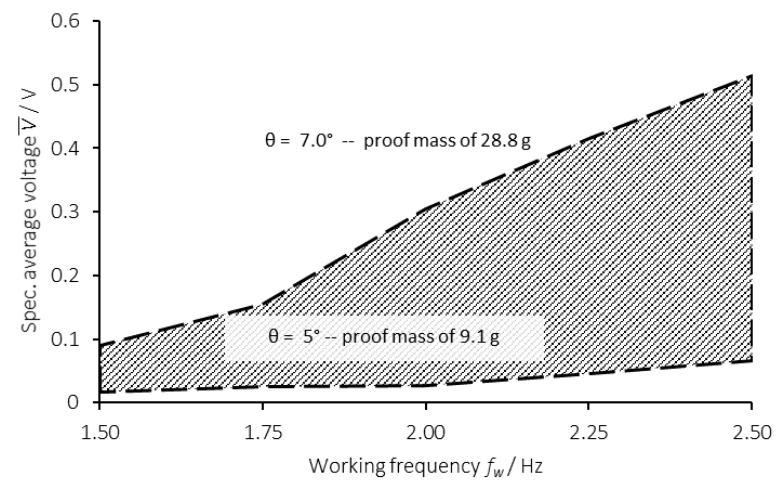

Figure 9. Specific average voltages $\bar{V}$ by the PEH obtained with respect the working frequency $f_{w}$.

change in the frequency of the DFT, but it acts on the amplitude of $V_{P E H}$. In fact, a greater proof mass induces a consequent increase in the DFT magnitude and a modification of the modal eigenvalues associated with the motion. Moreover, in these cases, the accelerations due to the proof masses assumed values between $0.77 \mathrm{~ms}^{-2}$ and $2.99 \mathrm{~ms}^{-2}$, calculated considering the parameters from Table 1 and Table 2.

Figure 7 presents the frequency effect of the imposed rolling motion on the harvested voltage $V_{P E H}$ with an angular displacement $\theta$ of $6.6^{\circ}$ and a proof mass of $24.1 \mathrm{~g}$. The frequency variation of the imposed rolling motion involves a shift of the frequency, but not an alteration in the magnitude of the harvested voltage. Specifically, the components of higher order are characterized by a greater frequency difference than those of lower order.

Figure 8 compares the frequency amplification factors of the buoy-PEH system as the angular displacement $\theta$ and working frequency $f_{w}$ of the imposed alternative rotational motion vary, using a proof mass of $24.1 \mathrm{~g}$. The amplification factors were obtained from the ratio between the frequency of the principal component of the harvested voltage and the working frequency $f_{w}$ of the imposed rolling motion. It was found that these factors are not very sensitive to either the frequency or the amplitude of the imposed rolling motion.

\subsection{Power estimation of the PEH}

Figure 9 shows the area that collects the values of the specific average voltages $\bar{V}$, calculated according to equation (8), as the working frequency $f_{w}$ varies. This area was obtained by changing the angular displacement $\theta$ of the boy and the proof masses glued to the PEH. The results are in perfect agreement with the literature [17]-[23]. In fact, it can be noted that as the proof mass increases (i. e. as the resonant frequency of the PEH decreases), a greater oscillation of the PEH is obtained with a consequent increment in the harvested voltage. A similar observation can be made by considering the increase in the working frequency $f_{w}$ of the imposed rolling motion with the same proof mass.

Figure 10 underlines the trend of the specific power $\bar{P}$, harvested by the PEH and calculated according to equation (9), as the working frequency $f_{w}$ and the amplitude of the angular displacement $\theta$ vary and using a fixed proof mass of $24.1 \mathrm{~g}$. A relative maximum is obtained at the working frequency of 2.25 $\mathrm{Hz}$ and its amplitude rises as the angular displacement $\theta$ of the buoy increases. This result also conforms to the data already present in the literature [17]-[23]. In the optimal conditions of a working frequency of $2.25 \mathrm{~Hz}$, an angular displacement of $7.0^{\circ}$ 


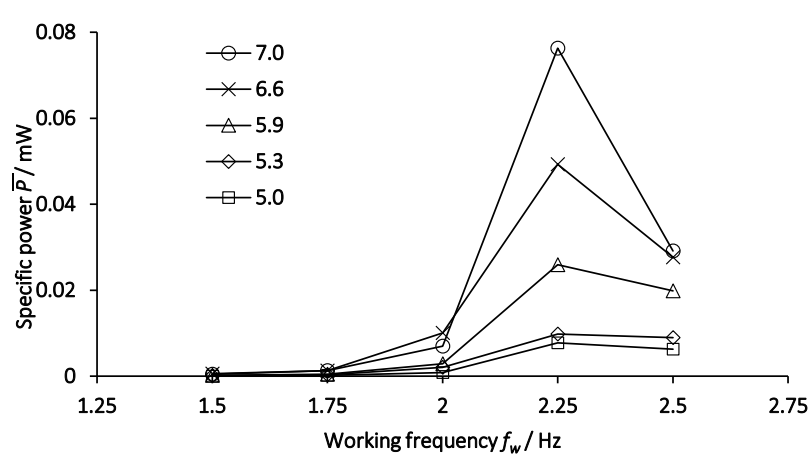

Figure 10. Specific power $\bar{P}$, harvested by the $\mathrm{PEH}$, as a function of the working frequency $f_{w}$ and the angular displacement $\vartheta$ with a proof mass of $24.1 \mathrm{~g}$.
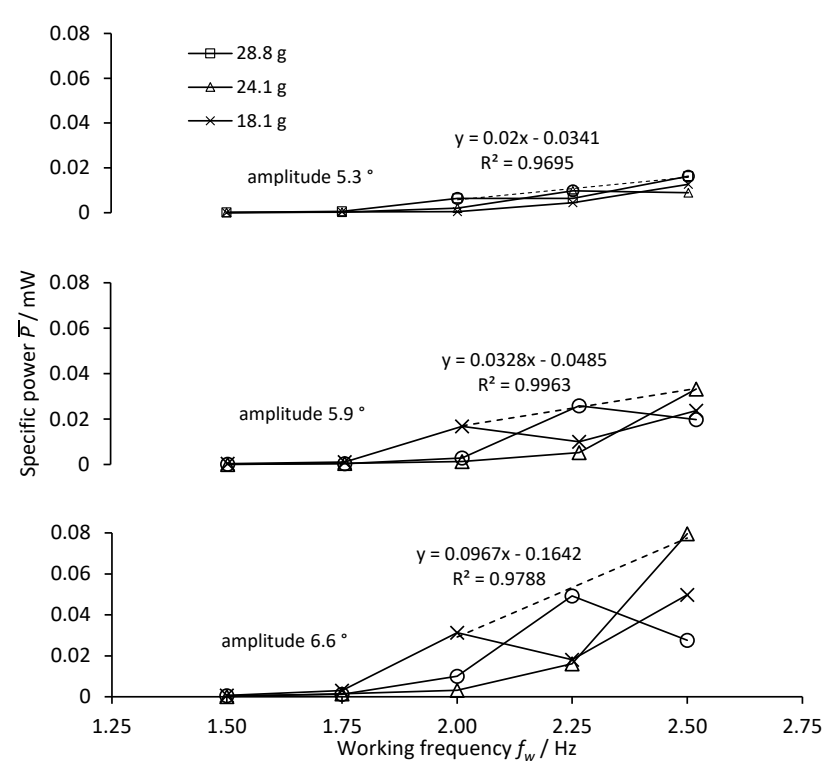

Figure 11 . Specific power $\bar{P}$, harvested by the $\mathrm{PEH}$, as a function of the working frequency $f_{w}$ and the proof mass at different angular displacement $\vartheta$

and a proof mass of $24.1 \mathrm{~g}$, the harvested energy in $1 \mathrm{~h}$ for a specific power of $0.08 \mathrm{~mW}$ is $274.6 \mathrm{mWh}$.

Figure 11 reports a comparison of the specific power $\bar{P}$, made with different proof masses and at the same angular displacement $\theta$. Because of that it is found in Figure 6, the different proof mass influences the harvested voltage $V_{P E H}$, and consequently the specific power $\bar{P}$, the latter proportional to the square of the voltage as visible in equation (9). As an example, Figure 12 exhibits the specific power $\bar{P}$ evaluated for the acquisitions with proof mass equal to 28.8 g. $\bar{P}$ assumes two relative maxima corresponding to the working frequencies $f_{w}$ equal to $2 \mathrm{~Hz}$ and $2.5 \mathrm{~Hz}$.

Generally, harvesters using piezoelectric patches in cantilever configuration are able to produce a power peak at its resonant

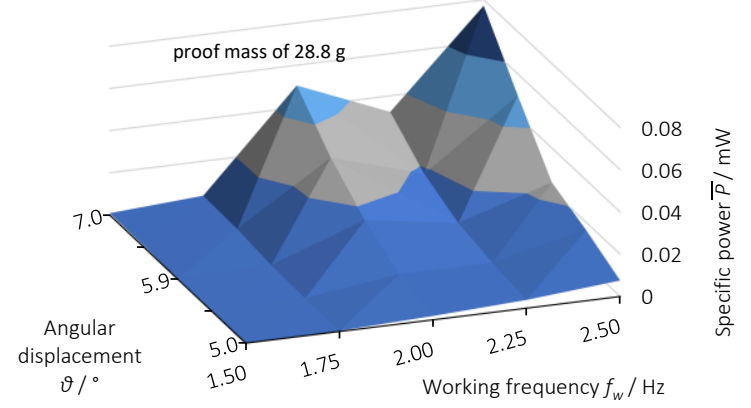

Figure 12. Specific power $\bar{P}$, harvested by the $\mathrm{PEH}$, for angular displacement $\vartheta$ and working frequency $f_{w}$ with a proof mass of $28.8 \mathrm{~g}$.

frequencies. It has already been observed that the first mode of vibration, usually at low frequencies, is responsible for the greater energetic contribution as it provides the components with the highest rate of deformation compared to the other modes of vibration [16]-[20].

\subsection{Uncertainty evaluation of the harvested power by the PEH}

The uncertainty of specific power $\bar{P}$ by the PEH is estimated by analysing the relative weight of each of the quantities in equation (9). The estimation of the combined uncertainty was based on the ISO/IEC Guide 98-3:2008 [34], by applying the following propagation law:

$$
u(y)=\sqrt{\sum_{i} u^{2}\left(x_{i}\right)\left(\frac{\delta \bar{y}}{\delta x_{i}}\right)^{2}} .
$$

The previous is explicated as:

$$
\begin{aligned}
u(\bar{P})= & {\left[u^{2}\left(f_{w}\right)\left(\frac{\delta \bar{P}}{\delta f_{w}}\right)^{2}+u^{2}(R)\left(\frac{\delta \bar{P}}{\delta R}\right)^{2}\right.} \\
& \left.+u^{2}(\bar{V})\left(\frac{\delta \bar{P}}{\delta \bar{V}}\right)^{2}\right]^{0.5} .
\end{aligned}
$$

The extended uncertainty $\left(U_{c}\right)$ was then estimated by assuming a coverage factor $(k)$ equal to 2.57 , based on a $t$ distribution with five degrees of freedom at a confidence level of 98\%. The detailed computation is shown in Table 3. Similar results were obtained for other acquisitions at different motion parameters. Looking at the relative weight of the different quantities affecting the uncertainty, it can be highlighted that the main contribution derives from the specific average voltages $\bar{V}$, whereas the other quantities have a much lower influence.

\section{CONCLUSIONS}

For a measurements buoy, the power consumption mainly depends on the components that deal with data communication.

\begin{tabular}{|c|c|c|c|c|c|c|}
\hline Parameter & value & uncertainty type & $u\left(x_{i}\right)$ & $u^{2} \cdot\left(\delta P / \delta x_{i}\right)^{2}$ & $u(P)$ & $U_{c}(P)(k=2.57)$ \\
\hline $\bar{V} / \mathrm{V}$ & 0.3193 & $A$ & $2.85 \mathrm{E}-2$ & $4.80 \mathrm{E}-5$ & & \\
\hline$f_{w} / \mathrm{Hz}$ & 2.00 & $A$ & 5.57E-14 & $4.34 \mathrm{E}-9$ & $9.49 E-5$ & $2.44 \mathrm{E}-4$ \\
\hline$R / \Omega$ & $1 \mathrm{E}+6$ & $B$ & $1.50 \mathrm{E}+3$ & $8.88 \mathrm{E}-21$ & & \\
\hline
\end{tabular}
In fact, data collection does not take place continuously over

Table 3. Uncertainty evaluation at the working frequency $f_{w}$ equal $2 \mathrm{~Hz}$ with an angular displacement $\vartheta$ of $6.6^{\circ}$ and proof mass of $24.1 \mathrm{~g}$. 
time. Therefore, these devices do not need to be constantly active and can often be put into sleep mode and waked up at fixed time intervals or in response to some external event. Typically, the power consumption for such routines is on the order of a few $\mathrm{mW}$ and can be ensured by rechargeable batteries and appropriate power electronics downstream the harvesters [35].

In this work, a simple and innovative layout for a small selfpowered floating buoy, employed for environmental monitoring activities, has been presented. This device makes use of a PEH, consisted of a PC, set in a cantilever configuration and excited by the rolling motion of the ripple waves, as supply source. The voltage and the power response of the PEH has shown a particularly advantageous behavior because the imposed motion has excited the PC with a multi-frequency combination of vibration modes. A multiplication of the oscillation frequencies for the PEH has been found as the working frequency varies. In fact, although the frequencies of the harvested voltage and power do not match the resonant frequency of the used PEH and therefore the best deformation for the PC is not obtained [16][21], the calculated specific power has relative maxima with respect to the input parameters (i. e. the frequency and amplitude of ripple waves). In this setup configuration and in optimal conditions of a working frequency of $2.25 \mathrm{~Hz}$, an angular displacement of $7.0^{\circ}$ and a proof mass of $24.1 \mathrm{~g}$, the PEH had reached the harvested energy in $1 \mathrm{~h}$ of $274.6 \mathrm{mWh}$ for a specific value of harvested power equals to $0.08 \mathrm{~mW}$. In this way the described floating buoy assumes the configuration of a frequency transformer. For these reasons, the analyzed layout allows to considerably increase the power generated by a single PEH, compared to that obtainable in typical conditions [26], [27], especially if such harvesters are coupled to a suitable impedance matching circuit [22], [23].

Future purposes will be aimed to identify the optimal position of the PC on the beam for a more efficiently conversion of the mechanical energy provided by ripple waves, to evaluate the effect of the noise superimposed on the main motion of the buoy, to define the scalability of the buoy after an appropriate fluid-dynamic sizing, and finally to estimate the buoy performance in real conditions.

\section{REFERENCES}

[1] T. P. Bean, N. Greenwood, R. Beckett, L. Biermann, J. P. Bignell, J. L. Brant (+28 authors), A review of the tools used for marine monitoring in the UK: combining historic and contemporary methods with modeling and socioeconomics to fulfill legislative needs and scientific ambitions, Frontiers in Marine Science 4 (2017) n. 263 pp. $1-29$.

DOI: $10.3389 /$ fmars.2017.00263

[2] H. Kim, L. Mokdad, ,J. Ben-Othman, Designing UAV surveillance frameworks for smart city and extensive ocean with differential perspectives, IEEE Communications Magazine 56 (2018) pp. 98 104.

DOI: $10.1109 /$ MCOM.2018.1700444

[3] L. G. A. Barboza, A. Cózar, B. C. Gimenez, T. L. Barros, P. J. Kershaw, L. Guilhermino, Macroplastics pollution in the marine environment, World seas: An environmental evaluation, Academic Press (2019) pp. 305-328. DOI: $10.1016 /$ B978-0-12-805052-1.00019-X

[4] A. L. Sobisevich, D. A. Presnov, M. V. Agafonov, L. E. Sobisevich, New-generation autonomous geohydroacoustic ice buoy, Seismic Instruments 54 (2018) pp. 677-681.

DOI: $10.3103 / \mathrm{S} 0747923918060117$

[5] S. Savoca, G. Capillo, M. Mancuso, C. Faggio, G. Panarello, R. Crupi, M. Bonsignore, L. D’Urso, G. Compagnini, F. Neri, E. Fazio, T. Romeo, T. Bottari, N. Spanò, Detection of artificial cellulose microfibers in Boops boops from the northern coasts of Sicily (Central Mediterranean), Science of the Total Environment 691 (2019) pp. 455-465

DOI: $10.1016 /$ j.scitotenv.2019.07.148

[6] Z. Chenbing, W. Xinpeng, L. Xiyao, Z. Suoping, W. Haitao, A small buoy for flux measurement in air-sea boundary layer, Proc. of the 13th IEEE International Conference on Electronic Measurement \& Instruments, ICEMI 2017, 20-22 October 2017, Yangzhou, China. DOI: $10.1109 /$ ICEMI.2017.8265999

[7] X. Roset, E. Trullos, C. Artero-Delgado, J. Prat, J. Del Rio, I. Massana, M. Carbonell, G. Barco de la Torre, D. Mihai Toma, Real-Time Seismic Data from the Bottom Sea, Sensors 18 (2018) n. 1132

DOI: $10.3390 / \mathrm{s} 18041132$

[8] L. M. Tender, S. A. Gray, E. Groveman, D. A. Lowy, P. Kauffman, J. Melhado, J. Dobarro, The first demonstration of a microbial fuel cell as a viable power supply: powering a meteorological buoy, Journal of Power Sources 179 (2008) pp. 571-575.

DOI: $10.1016 /$ i.jpowsour.2007.12.123

[9] J. Chen, Y. Li, X. Zhang, Y. Ma, Simulation and Design of Solar Power System for Ocean Buoy, Journal of Physics: Conference Series, IOP Publishing, 1061 (2018) pp. 012018. DOI: $10.1088 / 1742-6596 / 1061 / 1 / 012018$

[10] C. Alippi, R. Camplani, C. Galperti, M. Roveri. A robust, adaptive, solar-powered wsn framework for aquatic environmental monitoring, Sensors Journal, IEEE, 11 (2011) pp. 45-55. DOI: 10.1109/JSEN.2010.2051539

[11] C. Albaladejo, F. Soto, R. Torres, P. Sanchez, Juan A. Lopez, A low-cost sensor buoy system for monitoring shallow marine environments, Sensors 12 (2012) pp. 9613-9634. DOI: $10.3390 / \mathrm{s} 120709613$

[12] L. B. Hormann, P. M. Glatz, C. Steger, R. Weiss, A wireless sensor node for river monitoring using msp430® and energy harvesting, Proc. of the $4^{\text {th }}$ Education and Research Conference, EDERC 2010, 1-2 December 2010, Nice, France, pp. 140-144.

[13] J. F. Manwell, J. G. McGowan, A. L. Rogers, Wind energy explained: theory, design and application, John Wiley \& Sons, 2010.

[14] J. Trevathan, R. Johnstone, T. Chiffings, I. Atkinson, N. Bergmann, W. Read, S. Theiss, T. Myers, T. Stevens, SEMAT the next generation of inexpensive marine environmental monitoring and measurement systems, Sensors 12 (2012) pp. 9711-9748. DOI: $10.3390 / \mathrm{s} 120709711$

[15] J. Falnes, A review of wave-energy extraction, Marine Structures 20 (2007) pp. 185-201. DOI: $10.1016 /$ i.marstruc.2007.09.001

[16] R. Pelc, R. M. Fujita, Renewable energy from the ocean. Marine Policy 26 (2002) pp. 471-479. DOI: $10.1016 /$ S0308-597X(02)00045-3

[17] C. Borzea, D. Comeagă, A. Stoicescu, C. Nechifor, Piezoelectric Harvester Performance Analysis for Vibrations Harnessing. UPB Scientific Bulletin, Series C Electrical Engineering and Computer Science 81 (2019) pp. 237-248.

[18] R. M. Toyabur, M. Salauddin, and J. Y. Park, Design and experiment of piezoelectric multimodal energy harvester for low frequency vibration, Ceram. Int. 43 (2017) pp. 675-681. DOI: $\underline{10.1016 / \text { i.ceramint.2017.05.257 }}$

[19] E. L. Pradeesh, S. Udhayakumar, Effect of placement of piezoelectric material and proof mass on the performance of piezoelectric energy harvester, Mech. Syst. Signal Process. 130 (2019) pp. 664-676. DOI: $10.1016 /$ i.ymssp.2019.05.044

[20] R. Montanini, A. Quattrocchi, Experimental characterization of cantilever-type piezoelectric generator operating at resonance for vibration energy harvesting, AIP Conf. Proc. 1740 (2016) n. 60003.

DOI: $\underline{10.1063 / 1.4952675}$ 
[21] A. Quattrocchi, F. Freni, R. Montanini, Power Conversion Efficiency of Cantilever-Type Vibration Energy Harvesters Based on Piezoceramic Films, IEEE Transactions on Instrumentation and Measurement 70 (2021) n. 1500109, pp.1-9. DOI: $10.1109 /$ TIM.2020.3026462

[22] S. De Caro, R. Montanini, S. Panarello, A. Quattrocchi, T. Scimone, A. Testa, A PZT-based energy harvester with working point optimization, Proc. of the 6th International Conference on Clean Electrical Power, ICCEP 2017, $27-29$ June 2017, Santa Margherita Ligure, Italy, pp. 699-704.

DOI: $10.1109 /$ ICCEP.2017.8004767

[23] A. Quattrocchi, R. Montanini, R., S. De Caro, S. Panarello, S Scimone, S. Foti, A. Testa, A New Approach for Impedance Tracking of Piezoelectric Vibration Energy Harvesters Based on a Zeta Converter. Sensors 20 (2020) n. 5862. DOI: $\underline{10.3390 / \mathrm{s} 20205862}$

[24] N. Wu, Q. Wang, X. Xie, Ocean wave energy harvesting with a piezoelectric coupled buoy structure, Applied Ocean Research 50 (2015) pp. 110-118.

DOI: $10.1016 /$ j.apor.2015.01.004

[25] S. F. Nabavi, A. Farshidianfar, A. Afsharfard, Novel piezoelectricbased ocean wave energy harvesting from offshore buoys. Applied Ocean Research 76 (2018) pp. 174-18.

DOI: $10.1016 /$ j.apor.2018.05.005

[26] D. Alizzio, M. Bonfanti, N. Donato, C. Faraci, G. M. Grasso, F. Lo Savio, R. Montanini, A. Quattrocchi, Design and Performance Evaluation of a "Fixed-Point" Spar Buoy Equipped with a Piezoelectric Energy Harvesting Unit for Floating Near-Shore Applications, Sensors 21 (2021) n. 1912. DOI: $10.3390 / \mathrm{s} 21051912$

[27] D. Alizzio, M. Bonfanti, N. Donato, C. Faraci, G. M. Grasso, F. Lo Savio, R. Montanini, A. Quattrocchi, Design and verification of a "Fixed-Point" spar buoy scale model for a "Lab on Sea" unit, Proc. of the 2020 IMEKO TC19 International Workshop on
Metrology for the Sea, IMEKO TC19, October 5-7, 2020 Naples, Italy, pp. 27-32. Online [Accessed 15 December 2021] https://www.imeko.org/publications/tc19-Metrosea2020/IMEKO-TC19-MetroSea-2020-11.pdf

[28] A. Quattrocchi, F. Freni, and R. Montanini, Self-heat generation of embedded piezoceramic patches used for fabrication of smart materials, Sens. Actuators A Phys. 280 (2018) pp. 513-520. DOI: $10.1016 /$ i.sna.2018.08.022

[29] S. Sternini, A. Quattrocchi, R. Montanini, A. Pau, F. L. di Scalea, A match coefficient approach for damage imaging in structural components by ultrasonic synthetic aperture focus, Procedia Eng. 199 (2017) pp. 1544-1549.

DOI: $10.1016 / j . p r o e n g .2017 .09 .503$

[30] A. Erturk, D.J. Inman, Piezoelectric Energy Harvesting, Wiley, United States, 2011 ISBN: 978-0-470-68254-8

[31] A. Erturk, D.J. Inman, On mechanical modeling of cantilevered piezoelectric vibration energy harvesters, J. Intell. Mater. Syst. Struct. 19 (2008).

[32] A. Amanci, F. Giraud, C. Giraud-Audine, M. Amberg, F. Dawson, B. Lemaire-Semail, Analysis of the energy harvesting performance of a piezoelectric bender outside its resonance, Sens. Actuators A: Phys. 17 (2014) 129-138.

[33] Y. C. Shu, I. C. Lien, Efficiency of energy conversion for a piezoelectric power harvesting system, J. Micromech. Microeng. 16 (2006) n. 11 pp. 2429-2438.

DOI: $10.1088 / 0960-1317 / 16 / 11 / 026$

[34] Uncertainty of Measurement_-Part 3: Guide to the Expression of Uncertainty in Measurement, document ISO/IEC Guide 983:2008, 2008

[35] J. M. Gilbert, F. Balouchi, Comparison of energy harvesting systems for wireless sensor networks. Int. J. Autom. Comput. 5 (2008), pp. 334-347

DOI: $10.1007 /$ s11633-008-0334-2 